\title{
Mathematical model and heuristics for maximizing reliability of parallel systems with alternatives and budget constraints
}

\author{
Namsu Ahn \\ Department of Machinery and Systems Engineering, KMA, Seoul 01805, Republic of Korea \\ *E-mail of the corresponding author: namsu.ahn@gmail.com
}

\begin{abstract}
Reliability is defined as the ability to perform a given function over a period of time in a particular environment. Designing problem of the system structure appears in many industries, and various reliability optimization issues arise. Especially in military defence area, it is very important to achieve maximum reliability under a given budget. In this study, maximum reliability optimization problem in parallel system, each component has several alternatives, and limited budget reliability optimization problem is considered. Objective function of the problem is expressed as a nonlinear function because it is a reliability of a parallel system. Therefore, it is generally known as difficult to obtain a global optimal solution. In this paper, we propose a heuristic methodology that solves the problem by using Combinatorial Bender's Cut. The results of experiments proved that the proposed algorithm showed good performance in terms of value of the obtained reliability and consumed CPU time.
\end{abstract}

Keywords : parallel system, reliability, nonlinear programming, heuristic

DOI: $10.7176 /$ IEL/10-2-07

Publication date: April $30^{\text {th }} 2020$

\section{Introduction}

Reliability is defined as ability of a system to perform a required function in a given environment (or condition) for a specific period of time (Kim and Jung 2017). In this case, maximizing reliability under a given budget by properly selecting components of the system is a common problem in many industries.

Problem of maximizing the reliability of the system which considers available resources (budget, volume, weight, etc.), is called a Reliability Optimization Problem (ROP), and huge number of researches has been conducted (Kuo and Wan 2007, Slotani 2014, Zoulfaghari et al. 2015).

Subject of this research is, arrangement of components is a parallel structure, each component has several alternatives, there is an upper limit on the total budget, and the objective function is maximizing the system reliability. The stated problem can be depicted as shown in Figure 1. 
When arrangement of components of the system is serial, the reliability of the system can be calculated as $R_{s}=\prod_{i=1}^{n} r_{i}$ (system is designed with $\mathrm{n}$ components, where the reliability of each component $i$ is $r_{i}$, and the reliability of the system is $R_{s}$ ). In parallel system, the reliability of the system which is composed of $\mathrm{n}$ components is represented as $R_{s}=1-\left(1-r_{1}\right)\left(1-r_{2}\right) \ldots\left(1-r_{n}\right)$.

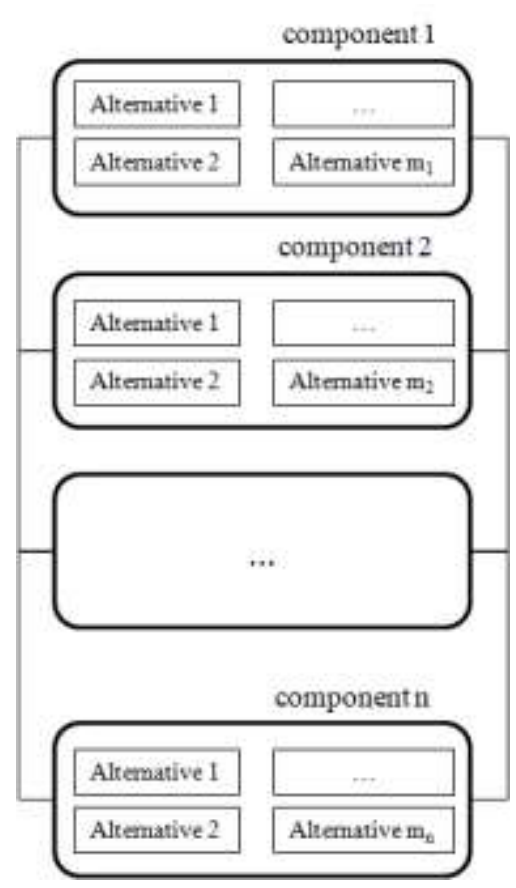

Figure 1. Parallel system which is composed of $\mathrm{n}$ components

This paper is organized as follows. Chapter 2 presents the nonlinear mathematical model to choose alternatives while maximizing Rs, and proposes an algorithm that can be used to solve the model by utilizing Combinatorial Bender's (CB) Cut. In the next chapter, the algorithm is implemented and computational results are summarized. The final chapter 4 introduces the conclusion of this study and suggests possible future research directions.

\section{Mathematical Model and Algorithm}

This chapter proposes a mathematical model for the ROP which was discussed in the previous chapter.

In here, we briefly review the symbols again. System consists of $\mathrm{n}$ components, and each component $i$ has mi alternatives. Total available budget is $C$, and cost and reliability of alternative $j$ for component $i$ are represented by $c_{i j}$ and $r_{i j}$, respectively. 
When the selection of alternative $j$ for component $i$ is represented as binary decision variable $x_{i j}$, the mathematical model can be given as follows.

$$
\begin{aligned}
& \max . R_{s}=1-\left(1-\sum_{j=1}^{m_{1}} r_{1 j} x_{1 j}\right)\left(1-\sum_{j=1}^{m_{2}} r_{2 j} x_{2 j}\right) \ldots\left(1-\sum_{j=1}^{m_{n}} r_{n j} x_{n j}\right) \\
& \sum_{i=1}^{n} \sum_{j=1}^{m_{i}} c_{i j} x_{i j} \leq C .(1) \\
& \sum_{j=1}^{m_{i}} x_{i j}=1, \forall i=1, \ldots, n .(2) \\
& x_{i j}=0 \text { or } 1, \forall i=1, \ldots, n, \forall j=1, \ldots, m_{i} .(3)
\end{aligned}
$$

Constraints (1) mean that the sum of the costs of the selected alternatives for all components cannot exceed the maximum available budget $(C)$. Constraints (2) indicate that the number of selected alternatives for each component must be exactly one. Constraints (3) show that all variables can only have a value of 0 or 1 . Lastly, objective function means maximizing the reliability of the parallel system.

However, since the above model is a nonlinear programming (NLP) model that includes a nonlinear objective function, it is known as not easy to obtain a global optimal solution or to prove obtained solution is a global optimal solution.

On the other hand, remaining constraints (1), (2) and (3) constitute the well-known Multiple Choice Knapsack Problem (MCKP) (Sinha and Zoltners 1979). Although the MCKP problem is known as the NP-complete problem, optimal solution can be obtained in a relatively short time (complexity of the algorithm is $O\left(\sum_{i} n_{i} \bar{b}\right), n_{i}$ and $\bar{b}$ represent the total number of alternatives and, respectively.).

In this study, we propose an algorithm that finds a solution by approximating a nonlinear objective function as a linear function $\left(=\sum_{i=1}^{n} \sum_{j=1}^{m_{i}} r_{i j} x_{i j}\right)$, and repeatedly solves the modified mathematical model until preset time is reached.

If the value of the modified objective function sets as $R s^{\prime}$, the heuristic algorithm can be expressed as Figure 2. 


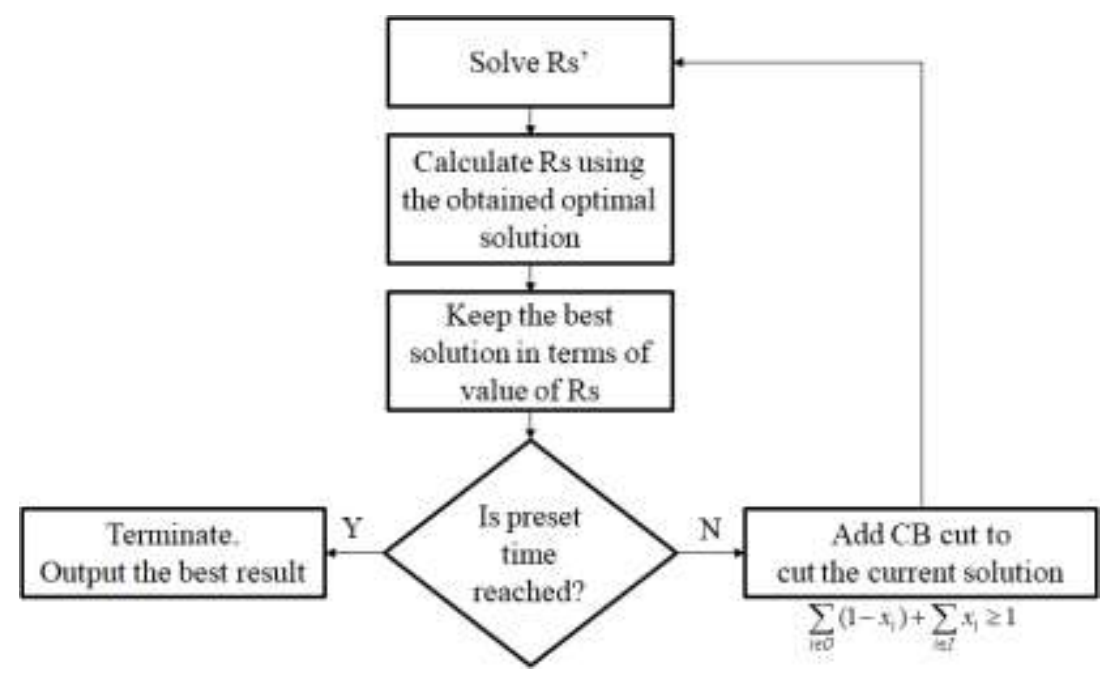

Figure 2. Flowchart of heuristic algorithm

After optimal solution $x^{*}$ of the modified problem is obtained, objective function value of the original problem can be calculated through $x^{*}$. Then, if the given time in advance is not exceed, the elements of $x^{*}$ whose values are 1 constitute set $\mathrm{I}$, and the elements of $x^{*}$ whose values are 0 constitute set $O$. Then, the following inequality (Combinatorial Bender's Cut) is added to the model to exclude the repetitive generation of $x^{*}$.

$$
\sum_{i \in I}\left(1-x_{i}^{*}\right)+\sum_{i \in O} x_{i}^{*} \geq 1
$$

Therefore, procedure of the algorithm can generate various solutions until a preset time is reached, and stores the value of the highest system reliability. When the preset time is reached, the algorithm outputs the best result.

\section{Computational Results}

In this chapter, we tried to verify the efficiency of the algorithm suggested in Chapter 2 to data of various scales. The experimental environment is, $\mathrm{CPU}$ i5 $1.6 \mathrm{GHz}$, Ram $8 \mathrm{~GB}$, and the algorithm was implemented using optimization software Xpress' Mosel (Dash 2020). For comparison purpose, the solution was compared using other optimization software Lingo (Lindo 2019), which is known to probably find a local optimal solution for a nonlinear mathematical model (Note that, Lingo may not be able to find an actual optimal solution even if it exists). 
Values of cost and reliability of alternative $j$ for component $i\left(c_{i j}\right.$ and $\left.r_{i j}\right)$ were generated using random numbers, respectively. However, if $r_{i j}$ value is too large, system reliability of the parallel system increases sharply and almost reaches to 1 . Therefore, those are adjusted to generate only values between 0 and 0.1 .

First, Tables 1 and 2 compare solutions when the numbers of components and alternatives are relatively small. Heuristic was terminated after 5 seconds (preset time), and in the case of Lingo, the time taken to find a solution was within 1 second. The last comparison column in Tables 1, 2 and 3 shows the comparison result of the obtained $R_{S}$ from heuristic and Lingo.

Table 1. Comparison of obtained $R_{s}$ when $n=5$

\begin{tabular}{|c|c|c|c|}
\hline \multirow{2}{*}{$\begin{array}{c}\text { Number of } \\
\text { alternatives }\end{array}$} & Heuristic & Lingo & Comparison \\
\cline { 2 - 4 } & \multicolumn{2}{|c|}{ Obtained $R_{s}$} & result \\
\hline 2 & 0.354405 & 0.2808424 & $>$ \\
\hline 3 & 0.251215 & 0.2512152 & $=$ \\
\hline 4 & 0.320411 & 0.3204114 & $=$ \\
\hline 5 & 0.31307 & 0.3130702 & $=$ \\
\hline
\end{tabular}

Table 2. Comparison of obtained $R_{s}$ when $n=10$

\begin{tabular}{|c|c|c|c|}
\hline \multirow{2}{*}{$\begin{array}{c}\text { Number of } \\
\text { alternatives }\end{array}$} & Heuristic & Lingo & Comparison \\
\cline { 2 - 4 } result \\
\hline 2 & 0.369432 & 0.3674931 & $>$ \\
\hline 3 & 0.54328 & 0.5432802 & $=$ \\
\hline 4 & 0.565867 & 0.5658673 & $=$ \\
\hline 5 & 0.585976 & 0.5859761 & $=$ \\
\hline
\end{tabular}


When the number of alternatives is small, there are cases in which the heuristic proposed in this study performed better than Lingo. Finally, as shown in Table 3, the computational time which is required to find the solution was compared by increasing the number of components and alternatives.

Table 3. Comparison of obtained $R s$ when the number of alternatives is relatively large

\begin{tabular}{|c|c|c|c|c|}
\hline \multirow{2}{*}{$\begin{array}{c}\text { Numbers of } \\
\text { components } \\
\text { and alternatives }\end{array}$} & Heuristic & \multicolumn{2}{|c|}{ Lingo } & \multirow{2}{*}{$\begin{array}{c}\text { Comparison } \\
\text { result }\end{array}$} \\
\hline & \multicolumn{2}{|c|}{ Obtained $R_{s}$} & $\begin{array}{c}\text { Consumed } \\
\text { time }\end{array}$ & \\
\hline 10 & 0.608554 & 0.608554 & $<1$ & $=$ \\
\hline 20 & 0.86612 & 0.866120 & $<1$ & $=$ \\
\hline 30 & 0.952607 & 0.952607 & $<1$ & $=$ \\
\hline 40 & 0.983647 & 0.983647 & $<1$ & $=$ \\
\hline 50 & 0.994292 & 0.994292 & $<1$ & $=$ \\
\hline 100 & 0.999968 & 0.999968 & 11 & $=$ \\
\hline
\end{tabular}

Interestingly, as the number of alternatives increases in Lingo, the CPU time required to solve the solution increases rapidly.

\section{Conclusions and Future Researches}

In this paper, we proposed a mathematical model to maximize the reliability of the parallel system and a heuristic algorithm to solve the model. However, since the objective function of the mathematical model was a nonlinear function, we tried to find alternative solutions by replacing the original objective function with a linear function, and, continuously excluded the current solution through the Combinatorial Bender's cut. This process tried to find better solution in terms of original objective function value.

Experimental results through the change of various factor values proved that the algorithm proposed in this study was effective in terms of obtained $R_{s}$ and consumed CPU time. 
The future research direction is to expand reliability optimization problem in mixture system of serial and parallel components. Also, reliability optimization problems in $k$ out of $n$ system or stand by systems can be another good future research topic.

\section{Acknowledgements}

This study was conducted through the support by Hwarang Research Institute of Korea Military Academy.

\section{References}

Kim, J. H. and Jung, W. (2017), Reliability Engineering 2nd, Chung Moon Gak.

Kuo, W. and Wan, R. (2007), Recent Advances in Optimal Reliability Allocation, IEEE Trans2 actions on Systems, Man, and Cybernetics - Part A: Systems and Humans, 37(2), 143-156.

Soltani, R.(2014), Reliability optimization of binary state non-repairable systems: A state of the art survey, International Journal of Industrial Engineering Computations, 5(3), 339-364.

Zoulfaghari, H., Hamadani, A., and Ardakan, M.(2015), Multi-objective availability redundancy allocation problem for a system with repairable and non-repairable components, Decision Science Letters, 4(3), 289-302.

Sinha, P., Zoltners, A. A.(1979), The multiple-choice knapsack problem, Operational Research, 27(3), 503-515.

Dash Optimization (2020), "Xpress Mosel 4.8.4".

Lindo Systems Inc. (2019), "Lingo 18.0.56”. 